\title{
Advanced Learning and ICT: new teaching experiences in University setting
}

\author{
Fabio Bertarelli, Matteo Corradini, Giacomo Guaraldi, Sergio Fonda,Elisabetta \\ Genovese, ,Stefano Dall'Olio, \\ ${ }^{1}$ Università degli Studi di Modena e Reggio Emilia, Via Vignolese 905/b, \\ 41125 Modena, Italy \\ \{ fabio.bertarelli matteo.corradini, giacomo.guaraldi,sergio.fonda, elisabetta.genovese, \\ stefano.dallolio, ,\}@unimore.it,
}

\begin{abstract}
Usually the digital board is thought of as a tool that can only be used beneficially in the context of primary school, secondary school or in a situation of learning handicap. In this case study we want to highlight how the new tools can be used in more broad settings such as teaching in scientific and technical universities. The easy adoption of all useful software on the market to the use of these tools makes them an innovative element in the teaching techniques of the future The article explores the use of advanced innovative technology in the classroom for effective teaching and learning. The digital whiteboard tool is a flexible and powerful didactic instrument that can greatly enrich the experience of both the learner and the teacher. In this article will be presented one completed real case in Italy, a work in progress case in Kenya and future works.
\end{abstract}

Keywords: Digital whiteboard, advanced teaching tools, repository, disability.

\section{Introduction}

Until the early '70s the Modena school system has taken a different path than that which until then had been made for persons with disabilities. The goal was the full integration with the outside world to hospitals. At that time there were no technological devices as common now and everything was based on the inclusion of disabled people in the school with the assistance of support teachers to better facilitate easy insertion. From those years, from that first experience was then gradually passed to a process of integration standardized.

The current computer technology have enabled the spread of innovative tools for the disabled.

Not that the history of pedagogy and psychology does had spoken of learning machines or tools to develop learning effectively, but until the advent of the current powerful and effective had not come to attribute to such subsidies such an important role in the dynamics teaching-learning. Back in the '50s, in fact Skinner (1954-1958) 
stated that if the computers were less expensive and less complicated to use could make an essential contribution education.

To summarize, wanting to trace the history of the diverse applications of information technology in education there are three specific phases characterized by different modes of computer use as a tool to teach:

Computer as a teaching machine. In this phase the compute understood as an instrument to replace the teacher. The personal computer offers to the student information (knowledge) previously stored in a manner of presentation treated in detail. The itinerary is strictly instructional, introduced and is implemented through a flowchart. The function is to perform a check on the performance of the student. The program allows you to continue learning or to stop and repeat.

Computer as a machine for thinking. In the second phase, the computer is seen and is the "thinking machine" for excellence. This vision is dictated by the need to remove the student from the passive position where it may find themselves in relation to the car. It is considered important, in other words, the student uses the computer, but also is seen as necessary to study computer science (programming languages) from the first steps of his training. Here then is the introduction of Logo in school and the Basic in high school. Later, through the development of application software packages (word processing, spreadsheets, databases etc. ..) will abandoned the study of languages and is passed to the teaching of the basics to get to the construction of hypertext.

Computer as a tool to communicate. The third phase is characterized by the spread of multimedia and the Internet. Here the computer is the tool of choice for entering into a relationship and acquire information. The network offers the opportunity to interact with the most diverse realities, with new ways to explore and "build". The new philosophy is simplicity of approach by facilitating interface, multimedia, the hypermediality and network, which now characterizes an almost wholly on software applications for teaching..

The advent of computer networks and the spread of software interfaces and ways to use strongly simplified and versatile [1] has done the rest.

In Modena, and elsewhere [2] from the mid-80s there was a gradual emergence of new tools and methodologies in order to use of personal computers in teaching and learning

it is on these assumptions, the boundary of this situation, with the same initial idea, that the University of Modena and Reggio Emilia decided to present a project then funded, introduction of the digital whiteboard in the teaching of academic disciplines.

The "interactive whiteboards at the University of Modena and Reggio Emilia" funded by MIUR (Ministero dell'Innovazione, Università e Ricerca), began in 2008, when the Disabled Student Services Home University has decided to implement a widespread implementation of digital whiteboard technologies within the University to fulfill what are its tasks, namely the inclusion of persons with disabilities and 
school (DSA) and, where possible, reducing disability due to modern technology (fig 1 and 2).

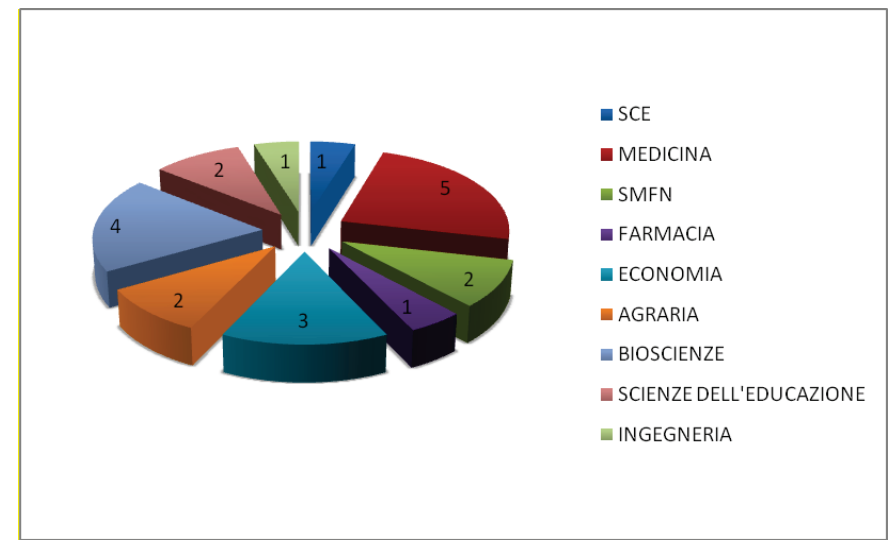

Fig. 1 Breakdown of students with special needs at the University of Modena and Reggio Emilia.

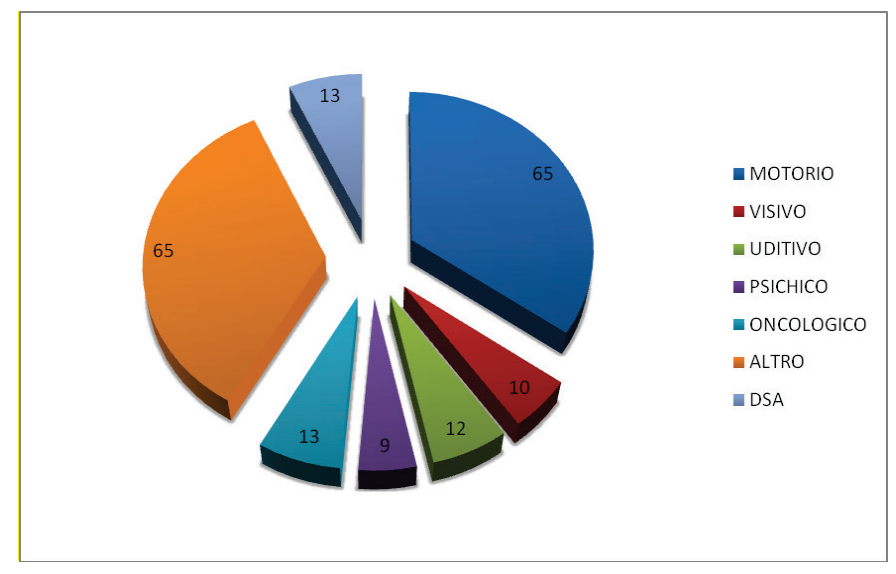

Fig. 2 Breakdown of students with disabilities enrolled at the University of Modena and Reggio Emilia.

\section{The Project}

The initial aim of this project was to show the advantages of adopting a new set of tools for classroom teaching, in particular, teachers explore the use of interactive whiteboards and associated software to evaluate the true effectiveness in terms of 
improvement of training specifically aimed at students with disabilities. The number of students attended to by the University Disability Service of the University of Modena and Reggio Emilia is equal to about 1\% of total students enrolled, which makes it even more significant when compared to the national average of $0.5 \%$. This fact was also an incentive for trying to increase the quality of service provided.

A further objective was to introduce a major technological innovation in the teaching of university courses, usually associated with fairly rigid patterns of presentation that hardly suit the needs of disabled students and more generally a little weak in supporting learning .

In particular it was decided to introduce new teaching aids such as digital whiteboards which can also store additional content during the frontal exposition by the teacher, store the teacher's classes, usually in Microsoft Power Point or noneditable PDF format.

The project was implemented according to previously established implementation steps and in particular:

1. Identification of appropriate technology.

2. Identification of the classrooms to equip ( 2 for each Faculty)

3. Drafting the contract

4. Construction of classrooms

5. Initial training targeted at each faculty with groups of up to 20 people

6. Continuous training based on the presence of certified internal trainers

7. Creation of an automatic repository for distribution of material to facilitate the distribution of digital lessons. (Developed by the Computing Center of the University)

8. Direct control of the distribution policy and manual submission of the material in the classroom by the teacher in order to maintain control over what you want to distribute

9. Export this experience to other universities or contexts, in order to increase understanding about the benefits in more real life cases

The first stages was reached by a close collaboration between Faculty Teaching Coordinators, Disability Services Office for disabled students, and technical staff responsible for supporting new technologies in order to identify classrooms to equip according to the usage and the practicability of an installation that is non-invasive to traditional teaching. The goal was to introduce a new method and not to replace one considered obsolete by some and yet for many still much more effective.

It was thus decided to use both by placing the digital whiteboard next to the standard board to allow gradual use.

To avoid the setting up of the PC for each use, the machines were installed in the vicinity of the digital whiteboard, wall mounted in armored cages without adding peripherals such as mice or keyboards. If need be, wireless keyboard and mouse could be used.

The decision to put two digital boards in the same faculty was made because of the following purposes: 
i. Fault Tolerance:- To have continuous operation in event one encountered technical problems (that has not occurred since).

ii. To encourage the use of the tool among teachers:- It is known that in the introduction of new technology there is always an initial exploratory phase in which the first steps are made with a little fear into the tool, and then begins a stage more "competitive" between the instrument and its user. This was in addition to creating a desire to share the experience with colleagues as the stage of insertion is typically more delicate.

iii. Having two large enough classrooms with digital whiteboards enabled the sharing of these resources with no initial conflicts in timetabling between teachers involved and creating curiosity and interest to those who had not yet approached the instrument.

After the drafting of the contract, the Computer center immediately began the analysis and implementation of the Repository in order to have the application up and running at the same time that the classrooms were equipped and ready. The repository was structured to create a folder for each faculty and dividing the contents of each in subfolders, one for each course curriculum.

Access is via authentication using the credentials of the student. In this way a student has access only to the course materials related to their curriculum.

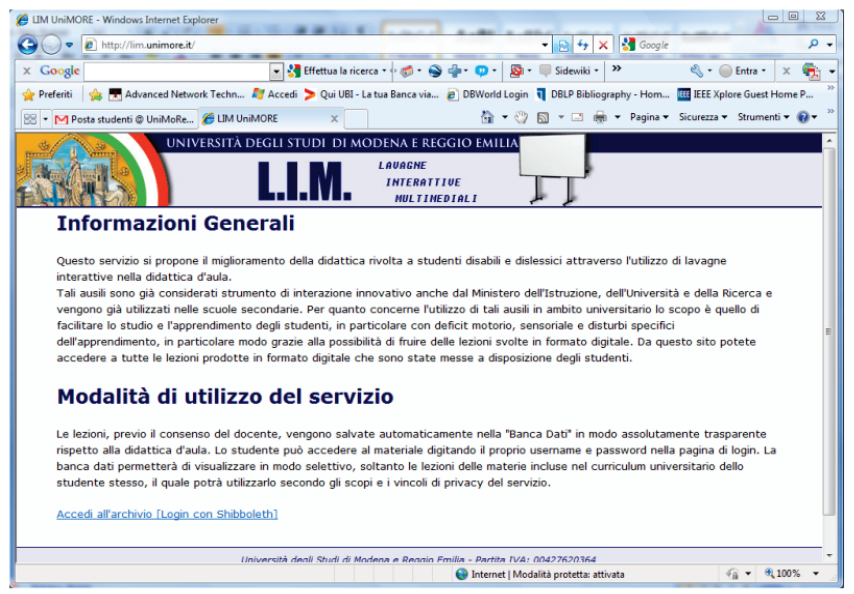

Fig. 3. Web page of the L.I.M Repository http://lim.unimore.it

To do that it was necessary to ascertain the specifications of the machine suitable for digital whiteboard and their location. Also it was necessary to ensure that no one 
could access the system without authorization, and that access to the new technologies does not require the assistance of a technician.

University technicians tied the use of the dedicated machine to the presence of a "certified" flash to the whole system's USB port. At the point of inserting the flash disk, the unit turns on automatically and the saving of the lessons presented in a specific folder either for publication or not are sent to it.

The idea of associating the serial number of the USB key (unique for each device produced in the world) was born to automate the writing and saving files in a simple way by the teacher and without adding information or work to the technicians to catalog all files in a second time before placing them in the database of lessons. On saving the lesson(s) on the network drive, the teacher has permission to publish the saved file directly in the folder of the course he/she teaches relevant to the rights they have, as explained in the description of the repository.

The identification of the user has an additional advantage in terms of transparency of the instrument: it is known that the PC market provides different operating systems, each of which must not be neglected to avoid having the initial disaffection towards the use of the instrument by the teacher.

To remedy this, the project was implemented using different virtual machines on an Apple system.

In accordance with the preference of the teacher (associated with the serial number of the key) an operating system is selected automatically at the start-up according to the request of the teacher certification made at the time of the key registration.

Enabling-disabling the key is the only operation required of the technician (one from each faculty) in addition to the normal operation of the equipment present. This happens in a few minutes using a simple program specifically implemented by the university's computer center.

\section{Inside the information system}




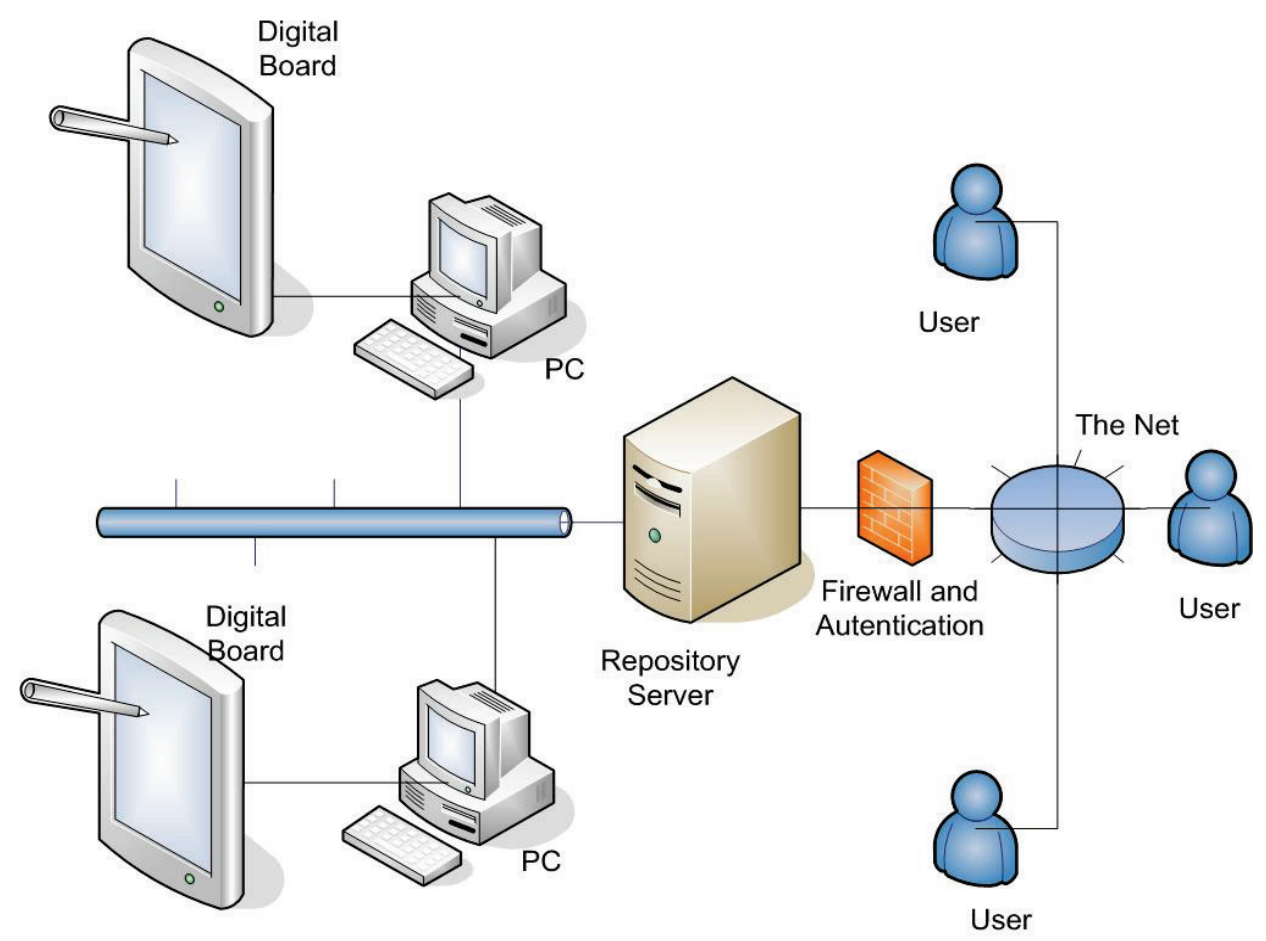

Fig. 4. Representation of the system devices connections : from Digital Board to the End Users

The overall system and in particular the Repository are born with two basic ideas:

1. to share with students the lessons that are created with the use of interactive whiteboards, and to simplify both the work of the teacher who then no longer have to carry files to specific teaching platforms (Moodle, or rather websites acts the function of the repository).

2. Being able to distribute free software to be deployed too high to allow universities to implement similar initiatives for the disability

The system is based on a web application interfaced with a system of SSO (Single Sign On), which regulates access.

Single sign-on is a specialized system that allows a user to authenticate once and access all computer resources to which it is enabled.

The reasons for this are several 
- simplify password management: the higher the number of passwords to manage, the greater the possibility that passwords will be used similar to each other and easy to store, thus lowering the level of safety;

- simplify the management of access to various services;

- simplify the definition and management of security policies.

Every student of the University has a serial number and a password that allows access to the information system may allow the use of all the enabled services at that 'user ID. This makes possible a certain selection ensuring student access only to files generated by the classes conducted in the faculty where he is registered, this guarantees the confidentiality of the files saved, so as to preserve as much as possible the intellectual property of teachers lessons.

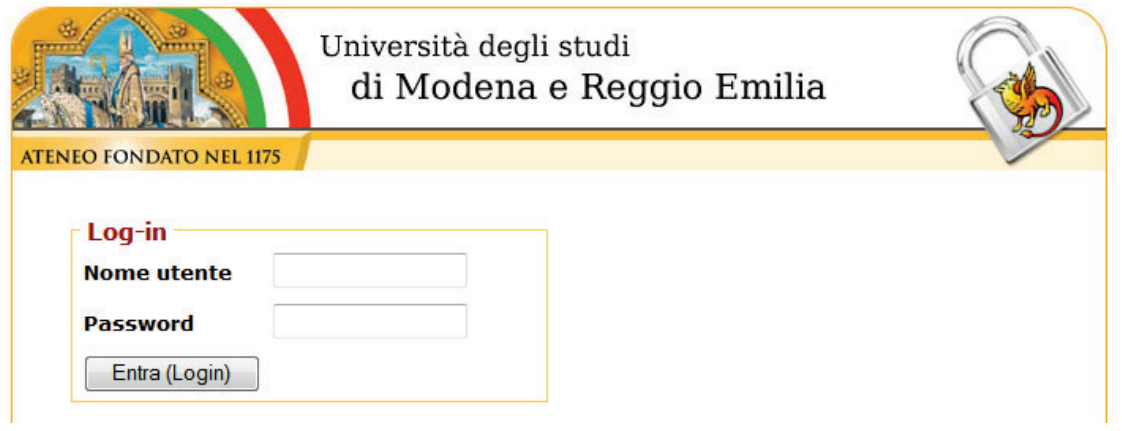

Fig. 5. Representation of the system devices connections : from Digital Board to the End Users

The system automatically and largely at night, download from computers connected to blackboards new files produced by the teacher during the use of blackboards digital files remain on the local computer until the end of the quarter so as to facilitate the teachers the conduct of teaching, if you encounter the need to recover even concepts not so temporally next. 


\begin{tabular}{|c|}
\hline File \\
\hline 1. Agraria \\
\hline 17. Bioscienze e Biotecnologie \\
\hline 13 Economia "Marco Biagi" \\
\hline 13 Farmacia \\
\hline Diurisprudenza \\
\hline Ingegneria (MO) \\
\hline Ingegneria (RE) \\
\hline (1) Lettere e Filosofia \\
\hline 17 Medicina e Chirurgia \\
\hline (1) Scienze della Comunicazione e dell'Economia \\
\hline 17 Scienze della Formazione \\
\hline 17. Scienze Matematiche Fisiche e Naturali \\
\hline D_Guest \\
\hline o Files - 13 Cartelle \\
\hline
\end{tabular}

Fig. 6. Representation of the system devices connections : from Digital Board to the End Users

The whole architecture of the repository system was developed in open-source and therefore has no maintenance cost and is written entirely in PHP with the advantage of being able to work on then-platform.

It is being developed distribution system multiforme (podcasting) of Materials (handouts, Notebook file, PDF files, audio-video, etc. ..) so as to allow the students themselves through the use of mobile systems, such as smartphones, netbooks, etc. ..

It was arranged the way to allow students to access in real time, on your computer, the lesson done by the teacher: in this way allows students to follow the hospitalization of distance learning courses with many advantages the terms of inclusiveness.

This latest technology is based on a system and a VNC on a transmission audio application "Skype"

The Virtual Network Computing (or VNC) is remote control software and are used to remotely administer your computer by installing a central system (in this case the $\mathrm{PC}$ in the classroom) and setting a proper password is allows others to get a VNC screen image and to send keyboard input and mouse to the computer server. In fact, 
you can manage your PC in the classroom if the teacher allows it, from another remote location as if it were their own computer.

L 'application "Skype" allows you to make phone calls completely free of charge, only works if both the sender and the recipient are connected to the internet and are linked through the Skype program that runs on your PC, and in this way it works is similar to any instant messaging systems such as ICQ or AIM or MSN Messenger, but it is always encrypted for security and privacy

These choices allow you to have simultaneously an open source system (the system VNC) for free and then install on the computers of students and a reliable and tested as Skype for voice transmission of the teacher.

The computer that runs locally digital whiteboard system is a MAC (minimac) running on a WindowsXP virtual machine preconfigured and reset when you start your computer, this allows the overall system more compatible and transparency by allowing the use by the teacher use the virtual machine Windows XP or Mac native system, in some cases at the specific request of teachers was also implemented a Linux virtual machine, and the teacher when the computer decides which of the operating system.

\section{Training}

Planning and training has been studied for the maximum effectiveness in the shortest possible time period chosen was close to the start of classes in order to encourage use since the start of courses.

The training was custom designed for each option so that only initial training was used to familiarize with the new tools and how they can publish the lessons. The rest was used to adapt innovative teaching to the needs of the teacher in explaining the topics. After an exploratory phase of the instrument was done to familiarize and techniques through learning-by-doing with the most valuable applications for the 'teaching of the interested parties, passing from the traditional to the one with standard software programs and then reach the 'use of multimedia such as movies or audio files

The lessons of 3 hours were structured in three phases:

An initial phase to become familiar with the tool. The description of the instrument was made based on the potential of the instrument, so as not to create the situation of waiting to new technologies, but as much as possible by facilitating the initial approach. It began using the digital whiteboard, as a real standard blackboard. The Collection of the Instruments available, such as pens of different stroke and color, geometric elements, marker and more, with a use very close to the use of the blackboard to which they were accustomed to the teachers, made a bridge to more advanced features.

She then emphasized the approach of the content on the screen, use of color and treatment, use of figures, for better appreciate the content of lessons. He directed the 
2011 Vol. 3 No. 4, pag. 377-388

DOI: http://dx.doi.org/10.1504/IJTEL.2011.041281

use of digital whiteboard towards creating storyboard to facilitate learning, rather than the classic use he sees as the result of a lesson, a single board with all the content while losing the chronological order of events.

At the end of this second phase, after about an hour and a half lesson as usual in all training courses, it is proposed in the working group or break. In almost all cases this situation has not only had the task to make a relaxing stop, but at this stage, some participants preferred to talk individually with the trainer asking for specific application solutions for their discipline. Practically all the meetings that have been made, the break rather than the ex-dividend basis, was a prelude to the final stage that applied to the discipline.

The initial idea of wanting to diversify the training depending on the faculty here has found confirmation of the validity of that choice.

Even for the trainer at this stage was full of ideas to better fit the tail of the first training meeting in this situation that saw the highest expression of innovation of teaching. From what has emerged in this last phase, and then think about how to set the work of post-training support team to better support and encourage the teachers more resourceful and support all those who have more timidly approached the instrument

\section{Innovation}

A strong innovative content was found when it was noticed that teachers would have wished in some cases to avoid using the digital whiteboard in the usual way or by means of presentation, going over to use the whiteboard linked to technical software like CAD or simulation programs by integrating the possibility to take visual notes, along with diagrams, and notes due to the flexibility that the digital whiteboard provides.

This combined extensive training offer of the University and the high level dellla audience consists of academics with a strong propensity for innovation in teaching have been an incentive for trainers. New testing techniques based on standard software with more specialized and dedicated not to teaching, but the study of individual disciplines. The teachers have taken quite happy to be able to comment directly on what they were presented, without having to go through other presentation tools. In particular, the image can pass the written form and the strong interaction that counts with a tool made it possible that at all stages, with particular emphasis during the exercises, the students (all students, not just those with special needs) are on, asking questions, technical questions, or bibliography, which often follow deepen not only verbal but also noting the specific contents are still present on the last page or previous pages of the blackboard An example can be seen in Figure 3 where the notes were made directly in the classroom during training and off-line. 


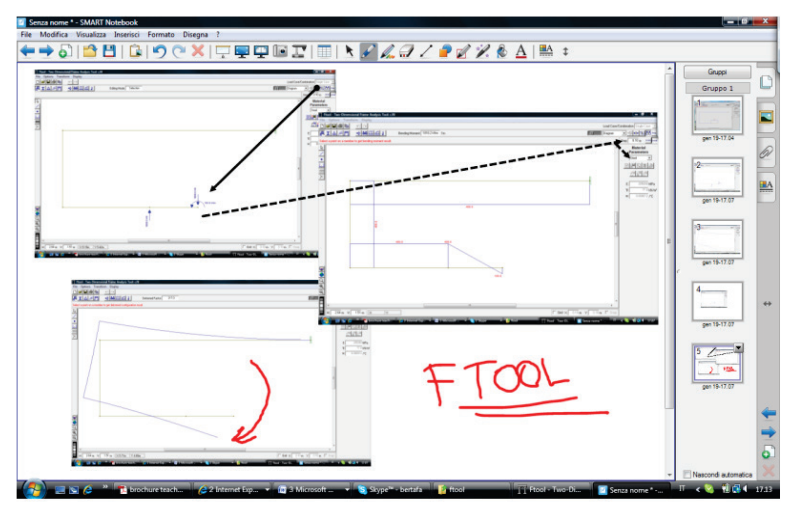

Fig. 3. Summary of a little storyboard of a specific engineering software (FTool). The digital whiteboard increases the teaching effectiveness and becomes an enhanced interface to explain and discuss with the classroom in an interactive mode about real cases and examples.

\section{Conclusion}

What had been thought for $1 \%$ of the students brought a benefit across the board. Several teachers have gradually begun to use new educational materials not only to improve the focus and effectiveness of instruction, but also stimulate a strong demand by students of the course material so obtained which allows a more complete reading of the topics addressed in the classroom. The increased motivation of students is palpable and some lessons on a trial basis were also made with audio support. All this was achieved without the presence of a technician in the classroom to support the new technology.

The introduction of new technologies in education requires a careful assessment of the impact it has on the teaching itself: the need for easy accessibility and easy use of new tools must take priority over everything. A teaching tool that is placed before the teacher that is invasive, impacts negatively on the performance of the teacher and hence on the quality of his teaching.

Finally, both the initial training that the subsequent training support for using advanced instrument should not be generalized but tailored to the needs and the use to which it is put. 


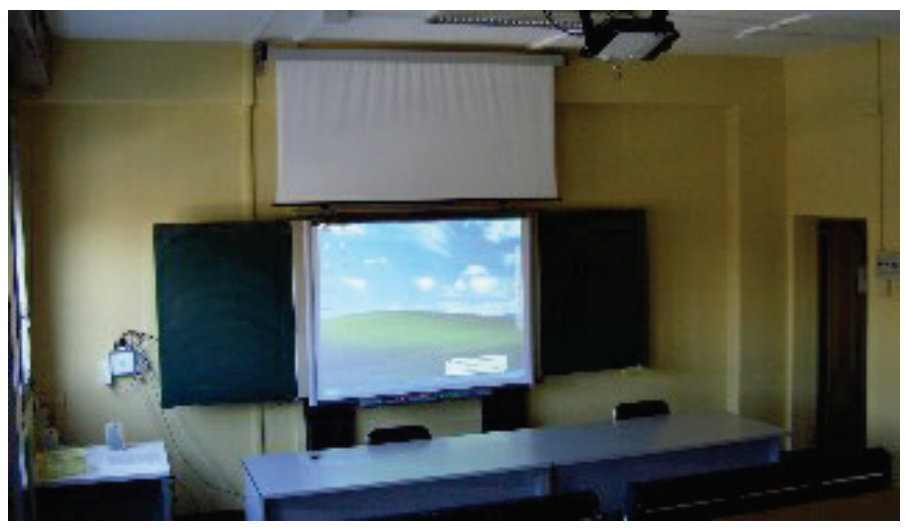

Fig. 4. A typical digital whiteboard installation: note the presence of the traditional blackboard and the installation of the PC fixed to the wall on the left

\section{Future work}

The passion and strong interest in new advanced techniques of instruction in addition to cooperation agreements between the University of Modena and Reggio Emilia and Masinde Muliro University of Science and Technology in Kenya, the staff of the two universities will evaluate the possible implementation of the project at the Kenyan university. In this case the benefit will be even greater as it will help reduce the cost in the use of white printable paper in this region. That can be avoided by sharing the lessons in softcopy by mailing list or USB flash disk.

In addition, Kenyan University registers a dismally low enrollment of the disabled pursuing higher education. This instrument might be used as a boost to having an improved presence if it is used across the board notwithstanding the level of learning (i.e. primary, secondary, tertiary) institution.

To make the fastest possible integration of the digital whiteboard in MMUST as expressed by their interest on the instrument, a staff member of Modena was hosted by the University in Kenya to find the best strategies and implementation modalities in the introduction of the new technologies

This is not the end point, although the results were encouraging. Several international research, have given prominence to new perspectives and new uses of ICT to help people with special needs.

The possibility of managing exams on-line [3] legally certifying the final results, make ICT a new tool to promote inclusion and disability compensation that places on an equal level with respect to what is required, restricting more and more the presence of tutors, which always create a sort of "diversification" in the administration of the tests 
The autonomy, the absence of other people, only using tools that are now in common use for all is a new step made to a society in this sense, less diverse and more uniform.

What Jackson Pollock said in his famous phrase "each age find its own tecnique", highlights groped as new roads such as the insertion into the world of work more productive and rewarding than just a few years ago. Experience of integration between universities and other productive sectors [4] and the search for new employment standards [5] are laying the foundations for a new phase of ICT usage and experience from which you started and we would be wrong if we are satisfied with what has been achieved, even if the students (disabled and not) and university professors welcomed the outcome of the project

\section{References}

[1] Application of word prediction and disambiguation to improve text entry for people with physical disabilities (assistive technology) - Hisham AlMubaid, Ping Chen-Int. J. of Social and Humanistic Computing 2008 Vol. 1, No.1 pp. $10-27$

[2] Integration of ICT into education in Slovak Republic - Ludmila Lubomir, Snajder Moravcikova, Ivan Kalas-Int. J. of Continuing Engineering Education and Lifelong Learning 2001 - Vol. 11, No.4/5/6 pp. 468-486

[3] Implementing digital signatures for healthcare enterprises: the case of online disability evaluation reports - Bengisu Tulu, Haiqing Li, Samir Chatterjee, Brian Hilton, Thomas Horan - Int. J. of Healthcare Technology and Management 2005 - Vol. 6, No.4/5/6 pp. 470 - 488

[4] University for Industry: widening participation? - Heidi R. Peel, Michael Quayle-Int. J. of Continuing Engineering Education and Lifelong Learning 2001 - Vol. 11, No.3 pp. 273-296

[5] Productivity and disability: the need to modify work standards - Anand Subramanian, Anil Mital - Int. J. of Productivity and Quality Management 2009 - Vol. 4, No.2 pp. 212 - 227 